\title{
Aqueous Chemical Solution Deposition of Functional Double Perovskite Epitaxial Thin Films
}

\author{
Hailin Wang ${ }^{[a]}$, Carlos Frontera ${ }^{[a]}$, Javier Herrero-Martín ${ }^{[b]}$, Alberto Pomar ${ }^{[a]}$, Pere Roura ${ }^{[c]}$, Benjamín \\ Martínez $^{*[a]}$, Narcis Mestres* ${ }^{[a]}$
}

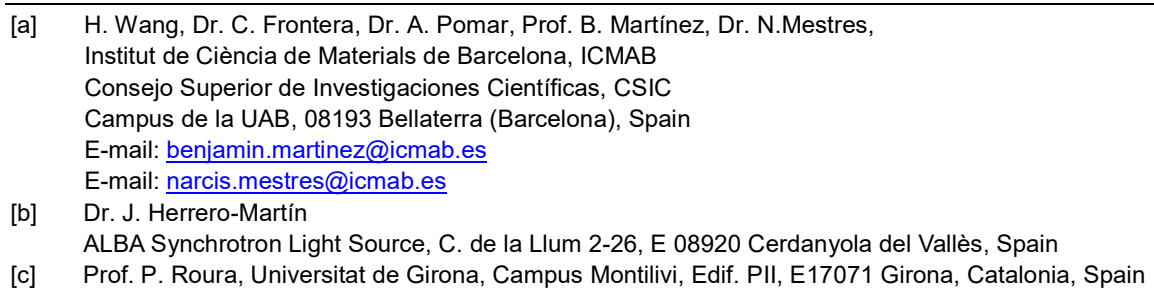

\begin{abstract}
:
Double perovskite structure $\left(\mathrm{A}_{2} \mathrm{BB}^{\prime} \mathrm{O}_{6}\right)$ oxides exhibit a breadth of multifunctional properties with a huge potential range of applications in fields as diverse as spintronics, magneto-optic devices or catalysis, and most of these applications require the use of thin films and heterostructures. Chemical solution deposition techniques are appearing as a very promising methodology to achieve epitaxial oxide thin films combining high performance with high throughput and low cost. In addition, physical properties of these materials are strongly dependent on the ordered arrangement of cations in the double perovskite structure. Thus, promoting spontaneous cationic ordering has become a very relevant issue. In this work, our recent achievements using polymer assisted deposition (PAD) of environmentally friendly, water-based solutions for the growth of epitaxial ferromagnetic insulating double perovskite $\mathrm{La}_{2} \mathrm{CoMnO}_{6}$ and $\mathrm{La}_{2} \mathrm{NiMnO}_{6}$ thin films on $\mathrm{SrTiO}_{3}$ and $\mathrm{LaAlO}_{3}$ single-crystal substrates are presented. We show that the particular crystallization and growth process conditions of PAD (very slow rate, close to thermodynamic equilibrium conditions) promote high crystallinity and quality of the films, as well as favors spontaneous B-site cationic ordering.
\end{abstract}




\section{INTRODUCTION:}

Complex oxides are a class of materials of strong technological interest because they not only present a broad variety of physical properties of technological interest but also several different mechanisms to modify these physical properties at convenience. In particular, the crystalline structure and aspects related to it (strain, defects, vacancies, cationic ordering,...) are an important playground to tune materials' properties. A common structure in oxide materials is the perovskite structure; ideal $\mathrm{ABO}_{3}$ perovskite structure has a cubic symmetry and most of the electronic properties are determined by the physics associated with the transition metal and the cornersharing oxygen anions of the $\mathrm{BO}_{6}$ octahedra. Double perovskite structure of the $\mathrm{A}_{2} \mathrm{BB}^{\prime} \mathrm{O}_{6}$-type, made of stacking single perovskite units, have attracted considerable attention because of their unique electrical, magnetic, and elastic properties. In the ideal double perovskite structure, in which $A$ is an alkaline earth or a rare-earth metal cation and $B$ and $B^{\prime}$ are transition metal cations, there exists a 3-D network of alternating $\mathrm{BO}_{6}$ and $\mathrm{B}^{\prime} \mathrm{O}_{6}$ octahedra. ${ }^{[1]}$ As a result of this, the final physical properties are strongly affected by the B-site cationic ordering. ${ }^{\left[{ }^{2]}\right.}$ Achieving full cationic ordering is challenging because the alternative occupancy of the $B / B^{\prime}$-site ions can be influenced by several factors, such as synthesis conditions and ionic features. As a general rule, a large difference in size and charge of the $B / B^{\prime}-$ site cations provides a material with higher degree of ordering. ${ }^{[1]}$ In particular, $\mathrm{La}_{2} \mathrm{MMnO}_{6}(\mathrm{M}=\mathrm{Co}, \mathrm{Ni})$ compounds with $\mathrm{B}$-site ordering display high Curie temperatures, ${ }^{[3]}$ which has been explained by superexchange interactions (FM-SE) according to the Goodenough-Kanamori rules. ${ }^{[4]}$ In addition, these oxides combine their high-temperature FM with insulating behavior. ${ }^{[5]}$ Dielectric properties close to room temperature ${ }^{[6]}$ and large magnetic field induced changes in the electric resistivity have also been reported. ${ }^{[7]}$ These properties make $\mathrm{La}_{2} \mathrm{MMnO}_{6}$ double perovskites interesting candidates for potential thin-film spin-based electronics, ${ }^{[8]}$ lead-free materials for use in solar cells ${ }^{[9]}$ and more recently, for cathode materials for intermediate temperature solid-oxide fuel cells. ${ }^{[10]}$ However, some of these properties critically depend on the degree of cationic ordering. In particular, the lack of cationic ordering results in competing ferro- and antiferromagnetic interactions and depressed saturation magnetization, ${ }^{\text {[5a] }}$ making the magnetic behavior a good indicator for the degree of cation ordering.

As oxides are increasingly applied as thin films or nanostructures to harness the varied benefits of miniaturization, convenient film deposition methods have to be developed. Although high-vacuum methods like molecular beam epitaxy, ${ }^{[11]}$ radiofrequency sputtering ${ }^{[12]}$ and pulsed laser deposition 
$(P L D)^{[13]}$ provide in general unquestionable advantages for the growth of metal oxide thin films including high crystal quality, precise control of composition and thickness at atomic-scale, more affordable alternatives are desirable. In this context, solution based fabrication methods are being pursued as an alternative for economically viable and large scale production of functional ceramic oxide thin films, nanoparticles, mesoporous solids films or bulk ceramics. ${ }^{[14]}$ The main advantage of this chemical solution deposition (CSD) methodology is the low cost, and easy scalability, associated to the process because the use of high vacuum systems is no longer required. In addition, the precursor solution can be modified in terms of solvent and molarity to tune different desirable thicknesses. Another advantage is the easy control of the desired final stoichiometry and the in-situ doping feasibility, simply by adding a doping agent to the precursor solution. The trend nowadays is the development of solution based processes that are environmental friendly, and processes that can be used with low temperature techniques such as self-combustion and deep-ultraviolet photochemical activation, enabling the direct integration of metal oxide layers on low melting point polymeric substrates for flexible electronic systems. ${ }^{[15]}$ The most recent achievements in this field show the successful integration of crystalline metal oxides on flexible substrates at temperatures as low as $350^{\circ} \mathrm{C}^{[16]}$

Concerning environmentally friendly precursor solutions, water is well recognized as an ideal and green solvent. However, with metal ions that are easy to hydrolyze (e.g., $\mathrm{La}^{3+}, \mathrm{Sr}^{2+}, \ldots$ ) it is difficult to form a stable solution in water. ${ }^{[17]}$ Moreover, it is hard to control the real metal ion concentration of the aqueous precursor solution, which often results in nonstoichiometric growth of the thin film compound. These problems have been solved by the approach proposed by Jia at al. ${ }^{[18]}$ preparing a stable metal-ion aqueous system, based on a homogeneous metal-polymer complex solution. A water-soluble polymer is used to bind and stabilize the metal ions in the precursor solution and at the same time facilitate the film coating. Specifically, the metal ions usually nitrate salts or chloride salts, are coordinated with lone-pair electrons of the nitrogen atoms in the polyethyleneimine (PEI) polymer, preventing in this way hydrolysis of metal ions and hence forming stable metal-polymer complexes. $^{[18-19]}$ In some cases, the use of an additional complexing agent like ethylenediaminetetraacetic acid (EDTA) is required to improve the coordination of metal cations to the polymer. To recapitulate, the PAD route is only composed of metal nitrates, multidentate polymers and deionized water, and consequently it is considered to be healthy and environmental friendly. Moreover, PAD solutions are highly stable over time and allow preparing a library of a 
large number of metal cations that can be subsequently mixed according to the desired stoichiometry. As a surfactant assisted synthesis method, the PEI present in the solution controls the viscosity and binds metal ions, resulting in an homogeneous mixing of metal ions in the gel and the formation of uniform metal-organic films, providing a facile chemical approach to both simple and complex crack-free epitaxial metal oxide films.

Despite the scientific and technological interest of $\mathrm{La}_{2} \mathrm{CoMnO}_{6}$ and $\mathrm{La}_{2} \mathrm{NiMnO}_{6}$ double perovskites, more effort is needed to bring them from laboratory research to applications. Towards this end, it is essential to search for synthesis routes able to provide thin films of target products with low cost and good control of composition, cation ordering, and cation charge and coupling.

In this context, the above mentioned specificities of PAD have proven to be very beneficial for the growth of complex oxide layers ${ }^{[20]}$ and recently, we have demonstrated the PAD growth of B-site ordered $\mathrm{La}_{2} \mathrm{CoMnO}_{6} / \mathrm{SrTiO}_{3}$ double perovskite epitaxial thin films, with perfect crystallinity and a high degree of B-site cationic ordering. ${ }^{[21]}$

In the present work we report on the epitaxial growth of $\mathrm{La}_{2} \mathrm{CoMnO}_{6}$ (LCMO) and $\mathrm{La}_{2} \mathrm{NiMnO}_{6}$ (LNMO) compounds on (001)- $\mathrm{SrTiO}_{3}$ (STO) and (001)- $\mathrm{LaAlO}_{3}$ (LAO) substrates by the PAD technique. We have investigated the influence of substrate, on the structural and magnetic properties of the films. LCMO epitaxial thin films exhibit a single magnetic phase with high saturation magnetization (Ms) values, indicative of full $\mathrm{Co}^{2+} / \mathrm{Mn}^{4+} \mathrm{B}$-site cationic ordering, whereas the LNMO films showed a more complex magnetic behavior suggesting the persistence of anti-site defects lowering $M_{s}$ with respect to the spin only maximum theoretical value.

\section{RESULTS and DISCUSSION}

To trace the thermal behavior of the mixed metal polymer precursor solution thin films during the annealing in a conventional tube furnace, we used thermogravimetric analysis combined with differential scanning calorimetry (TGA/DSC). Films of several hundreds of nanometers were obtained by the free spreading of a microdrop containing the precursor solution on glass substrates. High purity oxygen and synthetic air at a flow rate around $50 \mathrm{ml} / \mathrm{min}$ were used to control the furnace atmosphere with heating rate of $20^{\circ} \mathrm{C} / \mathrm{min}$. Figure 1 shows the TGA curve for a $\mathrm{La}_{2} \mathrm{NiMnO}_{6}$ precursor solution film. It can be seen that the sample begins to decompose at $220^{\circ} \mathrm{C}$, and that there is gradual mass loss (PEI, EDTA and nitrates loss) that stops at around $550{ }^{\circ} \mathrm{C}$. This 
suggests that the polymer and nitrate compound is completely decomposed and that the $\mathrm{La}_{2} \mathrm{NiMnO}_{6}$ phase may be obtained above this temperature. The heat of decomposition has been measured by DSC and an exothermic signal has been recorded with an exchanged heat of approximately $7.500 \mathrm{~J} / \mathrm{g}$.

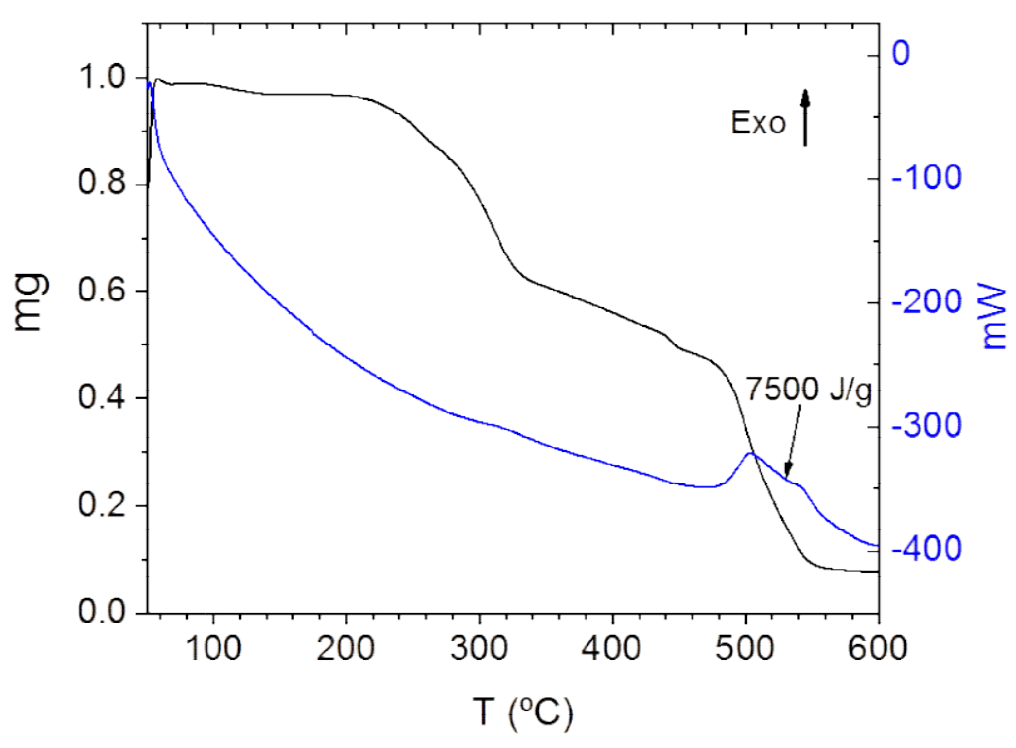

FIGURE 1. TG and DSC curves of the thermal decomposition of $\mathrm{La}_{2} \mathrm{NiMnO}_{6}$ precursors, made from nitrates precursors, EDTA and PEl.

In the PAD process, the coordination between the polymers and the metal cations prevents premature nucleation below the temperature of depolymerization of the polymers. In this way, the metal cations are inactive before the polymers are depolymerized. ${ }^{[19 a, 20]}$ Thus, crystallization of the oxide takes place near the decomposition temperature of the polymer and nitrates, i.e., close to thermodynamic equilibrium conditions; and it has been proposed that these particular growth conditions of PAD are much more favorable for attaining spontaneous B-site cationic ordering. ${ }^{[21]}$ Figure 2 shows AFM topography images in tapping mode of representative LCMO and LNMO films grown on top of STO and LAO substrates (film thickness $d \approx 25 \mathrm{~nm}$ ). Topography shows a high quality flat surface with root-mean-square ( $\mathrm{rms}$ ) values of surface roughness below $2 \mathrm{~nm}$ in all cases. For samples grown on STO substrates the RMS values is below $1 \mathrm{~nm}$. This fact may be related to the lower lattices mismatch between LCMO and LNMO and STO substrates, which favor a coherent growth (see below). Samples with thickness below $10 \mathrm{~nm}$ clearly show the terrace-like morphology imposed by the conformal growth on top of the substrate steps. ${ }^{[21]}$ 


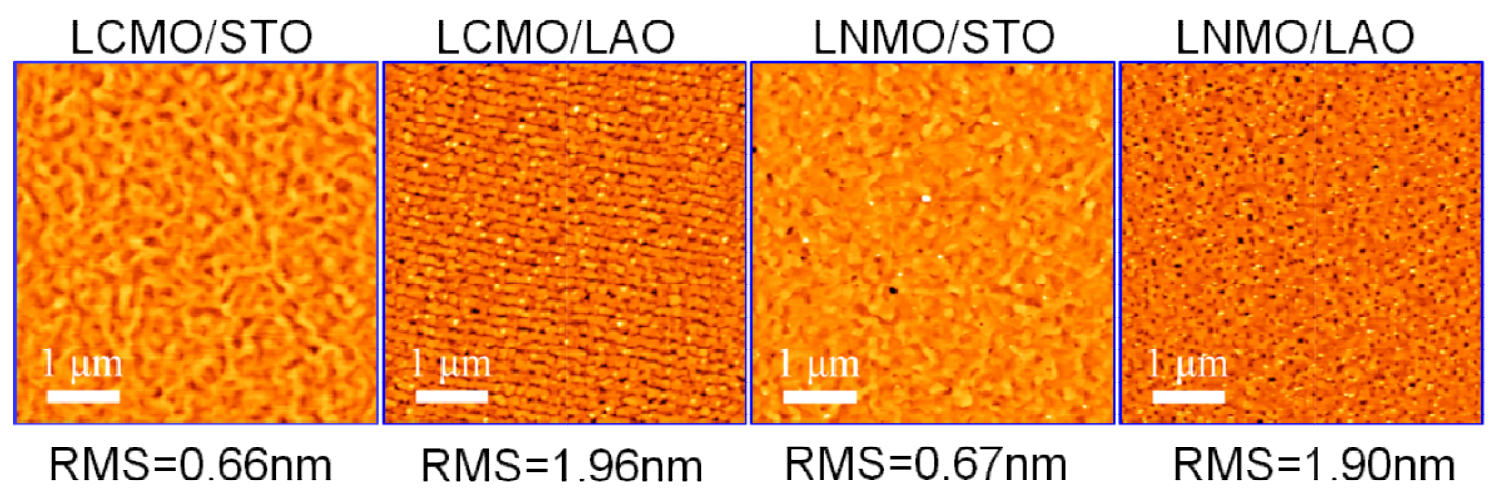

FIGURE 2. AFM surface topography images $\left(5 \times 5 \mu \mathrm{m}^{2}\right)$ of representative $\mathrm{La}_{2} \mathrm{MMnO}_{6}$ thin film samples with thickness values close to $25 \mathrm{~nm}$, on top of STO and LAO substrates. Root mean square (RMS) roughness values are below $2 \mathrm{~nm}$ in all cases.

The structural features of LCMO and LNMO films grown on STO and LAO substrates have been studied by x-ray diffraction. The epitaxial nature of the films was evidenced by the detection of only (0OI) peaks along with the corresponding (00I) peaks originating from the (001)-STO- and -LAO substrates, and the $K_{\beta}$ reflection.

Reciprocal space maps of LCMO films around (103) substrate peaks (Figs. 3(a) and 3(b)) reveal that the films on STO substrates grown fully strained without any measurable difference of the in-plane lattice parameters, as illustrated by Figure $3(\mathrm{a})$. An out-of-plane lattice parameter $c=3.872 \AA$ is estimated from the high-resolution X-ray diffraction pattern of the $(002)$ diffraction peak (see Fig. 3(c)). On the contrary, LCMO films grown on LAO were fully relaxed, and the in-plane parameter estimated from the reciprocal space map is about $a=3.89 \AA$, Figure $3(b)$. The high resolution $\theta / 2 \theta$ scan around the (002) substrate peak Fig. 3(d) was used to estimate an OP lattice parameter $c=3.886 \AA$. These values are consistent with a fully relaxed LCMO/LAO film.

(a)

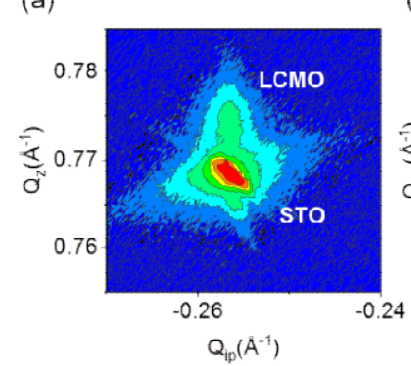

(b)

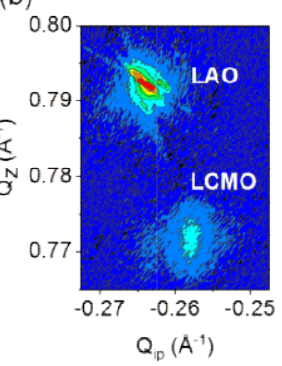

(c)

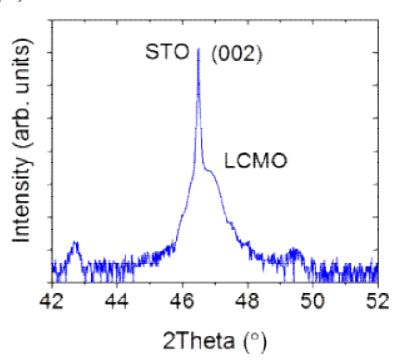

(d)

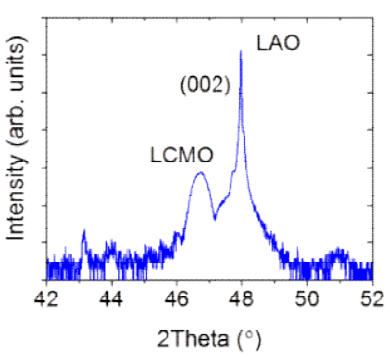

FIGURE 3: Reciprocal space maps around (103) reflections for LCMO films grown on STO (a) and LAO (b). (c) and (d). High-resolution $\theta / 2 \theta$ XRD scans of the (002) reflections for the same samples. 
A similar analysis was conducted on LNMO films. Reciprocal space maps of LNMO films around (103) substrate peaks (Figs. 4(a) and 4(b)) show that the films grown on STO substrates are fully strained (Fig. 4(a)). An out-of-plane lattice parameter $c=3.854 \AA$ is estimated from the highresolution X-ray diffraction pattern of the (002) diffraction peak (see Fig. 4(c)), which is shorter than the corresponding in plane lattice parameter. In contrast, the peak position of (103) reflection of the LNMO films grown on LAO is clearly shifted from that of the LAO substrate along the $Q_{100}$ axis Fig. 4(b). Although LNMO films are also grown epitaxially on LAO substrates, the large lattice mismatch $(-2.61 \%)$ between the films and the LAO substrate causes a relaxation of the films structure. In addition, the larger broadening of the LNMO film peak on LAO indicates that part of the sample is fully relaxed and some part is not.
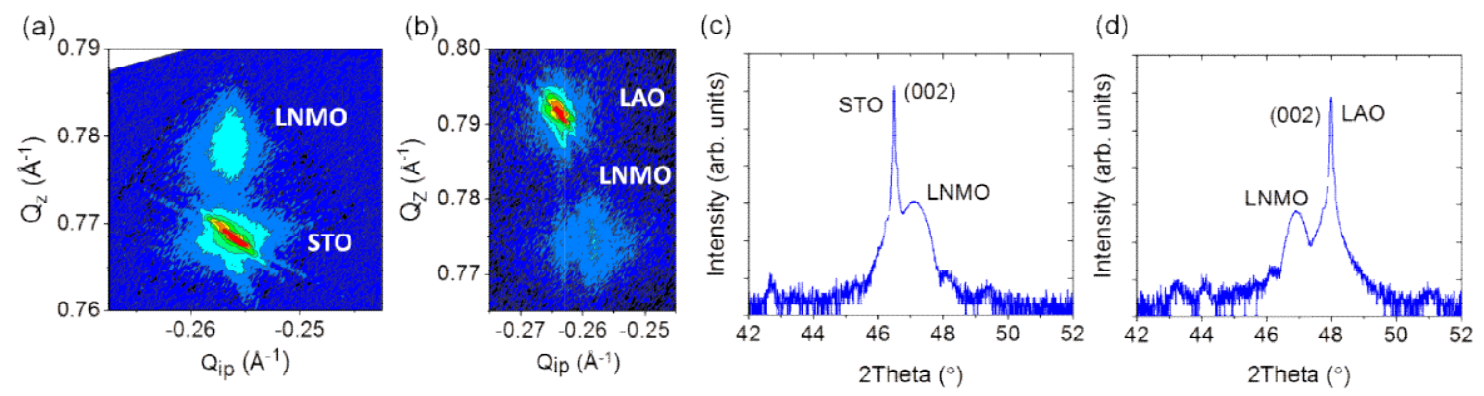

FIGURE 4. Reciprocal space maps around (103) reflections for LNMO films grown on STO (a) and LAO (b). (c) and (d) High-resolution $\theta / 2 \theta$ XRD scans of the (002) reflections for the same samples.

Since the lattice parameters of pseudocubic bulk $\mathrm{La}_{2} \mathrm{CoMnO}_{6}\left(a_{p c}=3.89 \AA\right)^{[22]}$ and $\mathrm{La}_{2} \mathrm{NiMnO}_{6}$ $\left(a_{p c}=3.879 \AA\right),{ }^{[23]}$ are close to the lattice parameters of STO substrate $(a=3.905 \AA)$, epitaxial $\mathrm{La}_{2} \mathrm{CoMnO}_{6}$ and $\mathrm{La}_{2} \mathrm{NiMnO}_{6}$ thin films grow coherently on this substrate. However, due to the large nominal lattice mismatch of the LCMO/LAO (-2.61\%) and LNMO/LAO (-2.31\%) systems, LCMO grows fully relaxed and LNMO partially relaxed on LAO substrates for film thickness $\approx 20 \mathrm{~nm}$. 


\section{MAGNETIC PROPERTIES}

\section{LCMO/STO}

Our Previous results in LCMO thin films prepared by sputtering indicate that irrespective to the structural strain (tensile or compressive) the oxidation states of $\mathrm{Co}$ and $\mathrm{Mn}$ ions are $\mathrm{Co}^{2+}$ and $\mathrm{Mn}^{4+} \cdot{ }^{[24]}$ According to the Goodenough-Kanamori rules, ${ }^{[4]}$ superexchange interaction between high spin $\mathrm{Co}^{2+}\left(3 d^{7}, t_{2 g}{ }^{5} e_{g}^{2}, S=3 / 2\right)$ and $\mathrm{Mn}^{4+}\left(3 d^{3}, t_{2 g}{ }^{3} e_{g}^{0}, S=3 / 2\right)$ cations promotes ferromagnetic (FM) ordering in fully ordered LCMO films. A Curie temperature, $T_{\mathrm{C}} \sim 230 \mathrm{~K}$, and a spin only theoretical saturation magnetization, $M s=6 \mu \mathrm{B} / \mathrm{f} . \mathrm{u}$. is observed. ${ }^{[5-6]}$ However, $\mathrm{Co}$ and $\mathrm{Mn}$ ions may interchange their crystallographic sites giving place to what is known as antisite disorder (ASD). Since ASD will promote the appearance of $\mathrm{Co}^{2+}-\mathrm{O}-\mathrm{Co}^{2+}$ and $\mathrm{Mn}^{4+}-\mathrm{O}-\mathrm{Mn}^{4+}$ antiferromagnetic (AFM) interactions the saturation magnetization, $M_{S}$, will be reduced by a factor (1-2X $\left.X_{A S D}\right)$ being $X_{A S D}$ the fraction of ASD disorder. ${ }^{[25]}$ Therefore, $M_{S}=\left(1-2 X_{A S D}\right) 6 \mu B / f . u$. is a very sensitive indicator of the degree of B-site cationic ordering. Recently, we have shown that the particular growth conditions of PAD (very slow rate and close to thermodynamic equilibrium conditions) are prone to promote high quality, as well as B-site ordered, LCMO/STO epitaxial thin films. ${ }^{[21]}$ Accordingly, the magnetic properties of a 26$\mathrm{nm}$-thick LCMO/STO thin film grown in optimized conditions $\left(900{ }^{\circ} \mathrm{C}\right.$ for $60 \mathrm{~min}$ with an oxygen flow of $0.3 \mathrm{l} / \mathrm{min}$ ) shown in Fig. 5, exhibit a Curie temperature $T c=230 \mathrm{~K}$ (see Fig. 5 (a)). Moreover, no signals for the coexistence of different FM phases, as usually occurs in oxygen-deficient samples prepared by PLD or sputtering ${ }^{[12 b, 26]}$ are detected. In Fig. 5(b), the measured low temperature (10 K) saturation magnetization Msat $\approx 5.9 \mu \mathrm{B} / \mathrm{f}$.u. and a coercive field of $\mathrm{Hc} \approx 10 \mathrm{kOe}$ for an external magnetic plane applied perpendicular to the substrate plane (OP-configuration) agree well with data reported for B-site ordered LCMO bulk ${ }^{[27]}$ and thin film samples. ${ }^{[12 b, 28]}$ 

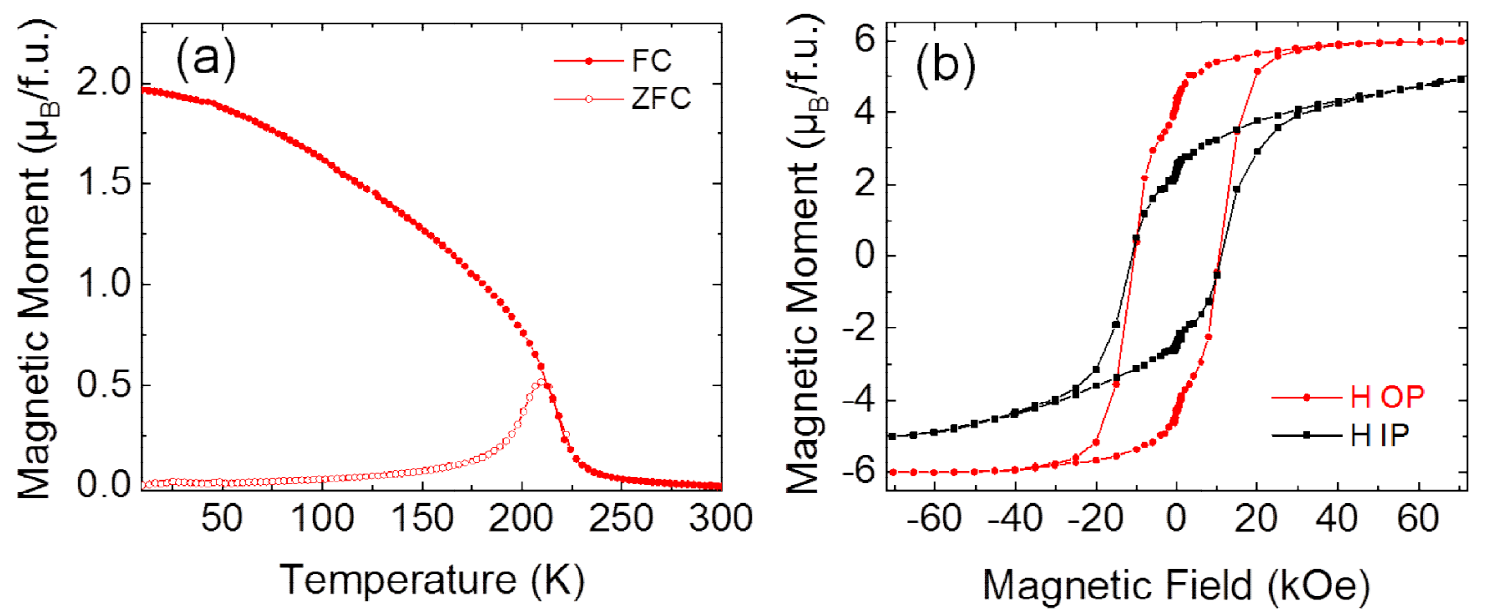

Figure 5. (a) Magnetization vs temperature of an LCMO/STO film of $26 \mathrm{~nm}$ grown at $900{ }^{\circ} \mathrm{C}$ measured after zero field cooling (open symbols) and field cooling (solid symbols) with a magnetic field of $1 \mathrm{kOe}$ applied inplane. (b) $\mathrm{M}-\mathrm{H}$ loops recorded at $10 \mathrm{~K}$ by applying a magnetic field parallel to (100)-STO (in-plane); and parallel to (001)-STO (out of plane).

Additionally, multiple nucleation sites can generate zones with alternated $\mathrm{Co} / \mathrm{Mn}$ local ordering. When two of these zones merge together deviations from the ideal $\mathrm{Co} / \mathrm{Mn}$ ordering appear at the interface giving place to $\mathrm{Co}^{2+}-\mathrm{O}-\mathrm{CO}^{2+}$ and $\mathrm{Mn}^{4+}-\mathrm{O}-\mathrm{Mn}^{4+} \mathrm{AFM}$ interactions generating an antiphase boundary (APB). APBs also promote a reduction of the saturation magnetization; however, their effect is very much smaller than that of ASD. They act as domain walls between the FM domains, within which a $\mathrm{Co}^{2+} / \mathrm{Mn}^{4+}$ ordering exists, ${ }^{[2 d, 4 b]}$ and their signature appears as a sudden drop in the remnant magnetization at $\mathrm{H}=0$, as observed in Figure $5(b) .{ }^{[5 a, 21]}$ This figure also makes evident the strong anisotropic behavior of the LCMO/STO thin film samples, being the OP direction the easy magnetization direction in agreement with previous work. ${ }^{[12 b, 24]}$

\section{LCMO/LAO}

Similar results are obtained for LCMO/LAO thin films (see Fig. 6). The temperature dependence of the magnetization for a $20 \mathrm{~nm}$ thick sample grown at a temperature of $900{ }^{\circ} \mathrm{C}$ with an oxygen flux of $0.3 \mathrm{I} / \mathrm{min}$, measured in IP configuration under a field of $1 \mathrm{kOe}$ is shown in Figure 6(a). A Curie temperature of $230 \mathrm{~K}$ was observed with no signals of any secondary phase, indicating optimum oxygen content and high film quality. 

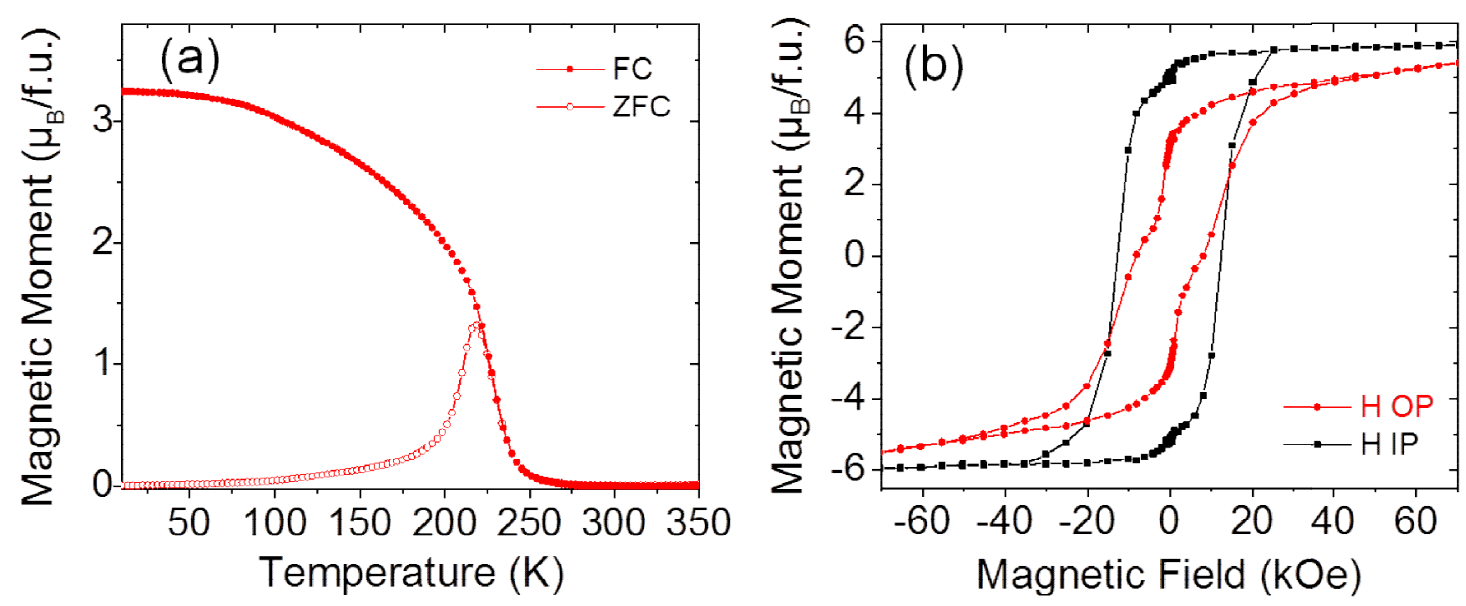

Figure 6. (a) Magnetization vs temperature of an LCMO/LAO film of $20 \mathrm{~nm}$ grown at $900{ }^{\circ} \mathrm{C}$ measured after zero field cooling (open symbols) and field cooling (solid symbols) with a magnetic field of $1 \mathrm{kOe}$ applied inplane. (b) $\mathrm{M}-\mathrm{H}$ loops recorded at $10 \mathrm{~K}$ by applying a magnetic field parallel to (100)-LAO (in-plane); and parallel to (001)-LAO (out of plane).

It is also evident from $M(H)$ curves in Figure $6(\mathrm{~b})$ that IP is the easy magnetization direction as expected for LCMO films under in-plane tensile strain. ${ }^{[24 b]}$ On the other hand, Fig. $6(\mathrm{~b})$ also shows that IP saturation magnetization almost reaches the spin only saturation value of $6 \mu_{\mathrm{B}} / \mathrm{f}$.u., therefore indicating full B-site cationic ordering as in the case of LCMO/STO films. As in the previous case, clear signatures of the existence of APB are also evident in Fig. 7(b).

\section{LNMO STO}

We move now to the analysis of the magnetic properties of LNMO samples. In contrast to the LCMO system, the $M(T)$ curves in LNMO samples exhibit a more complex structure that in some cases has been considered as indicative of the existence of different magnetic phases ${ }^{[29]}$ with two distinct magnetic transition temperatures at $T_{C 1} \approx 270 \mathrm{~K}$ and $T_{C 2} \approx 150 \mathrm{~K}$ (see Figure $7(\mathrm{a})$ ). The sharp transition at $T_{c 1} \approx 270 \mathrm{~K}$ was attributed to the $\mathrm{Ni}^{2+}-\mathrm{O}-\mathrm{Mn}^{4+}$ superexchange interaction between $\mathrm{Ni}^{2+}\left(d^{8}, \mathrm{t}_{2 \mathrm{~g}}{ }^{6} \mathrm{e}_{\mathrm{g}}{ }^{2}, \mathrm{~S}=1\right)$ and $\mathrm{Mn}^{4+}\left(\mathrm{d}^{3}, \mathrm{t}_{2 \mathrm{~g}}{ }^{3} \mathrm{e}_{\mathrm{g}}{ }^{0}, \mathrm{~S}=3 / 2\right)$ ions due to $\mathrm{Ni} / \mathrm{Mn}$ cation ordering. At the same time, the broad transition at $T_{c 2}$ about $150 \mathrm{~K}$ was ascribed to the $\mathrm{Ni}^{3+}-\mathrm{O}-\mathrm{Mn}^{3+}$ superexchange interaction between low-spin $\mathrm{Ni}^{3+}\left(d^{7}, \mathrm{t}_{2 g}{ }^{6} \mathrm{e}_{\mathrm{g}}{ }^{1}, \mathrm{~S}=1\right)$ and high-spin $\mathrm{Mn}^{3+}\left(\mathrm{d}^{4}, \mathrm{t}_{2 \mathrm{~g}}{ }^{3} \mathrm{e}_{\mathrm{g}}{ }^{1}\right.$, $S=3 / 2$ ) cations. ${ }^{\left[{ }^{30]}\right.}$ However, recent results indicate that this particular shape of the $M(T)$ curve is not indicative of different magnetic phases but might well be related with the existence of ASD (i.e. disordered occupancy of the Ni/Mn sites). ${ }^{[31]}$ Accordingly, featureless $M(T)$ curves should be indicative of a smaller amount of ASD (see Fig. 7(a)). Nevertheless, it is worth noting that $M(T)$ 
curves in $\mathrm{Ni} / \mathrm{Mn}$ system are always much flattened than those of $\mathrm{Co} / \mathrm{Mn}$ system which could be indicative of a higher degree of cationic disorder. This fact is also confirmed by a smaller saturation magnetization with respect to the spin only theoretical value of the $\mathrm{LNMO}$ system $(\mathrm{Ms}=5 \mu \mathrm{B} / \mathrm{f} . \mathrm{u})$ (see Fig. 7(b)). Figure 7(a) also evidences that FM transition temperature is $T_{c} \approx 270 \mathrm{~K}$, irrespective to the amount of ASD in the sample, since $T_{C}$ is proportional to the strength of the superexchange FM interactions that are stronger in the LNMO system than in LCMO.
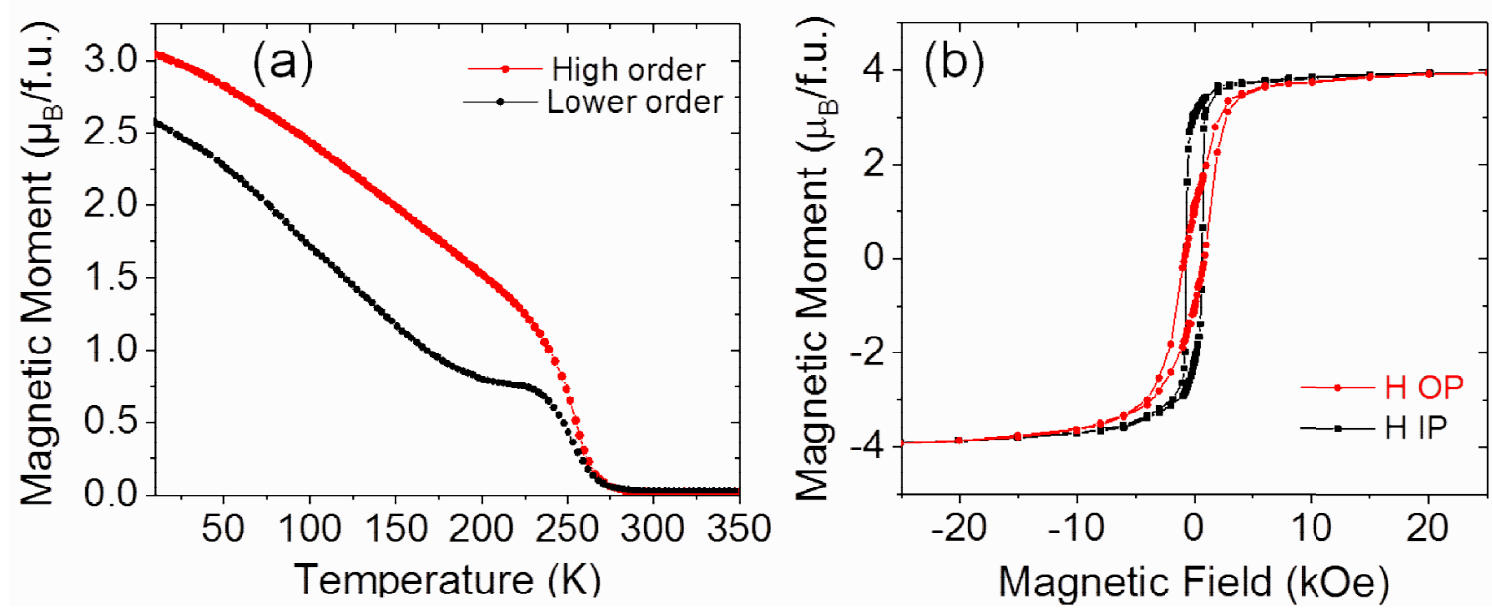

Figure 7: (a) Magnetization vs. temperature of two LNMO/STO films with different amount of ASD, measured after field cooling with a magnetic field of $1 \mathrm{kOe}$ applied in-plane. (b) $\mathrm{M}-\mathrm{H}$ loops of the more ordered LNMO/STO film in (a) recorded at $10 \mathrm{~K}$ by applying a magnetic field $\mathrm{H}$ parallel to (100)-STO, in-plane; and parallel to (001)-STO, out of plane.

\section{LNMO_LAO}

Similar results are obtained for LNMO films grown on top of LAO substrates (see Fig. 8(a)). M(T) curves are very much alike to the LNMO/STO samples with a FM transition temperature $T_{C} \approx 270 \mathrm{~K}$. As in the previous case, samples with larger amount of ASD exhibit a non-monotonic $M(T)$ curve with a local minimum around $T \approx 150 \mathrm{~K}$ (see Fig. $8(\mathrm{a})$ ). This is further supported by the smaller saturation magnetization values obtained for these samples (see Fig. 8(b)).

Comparison between LCMO and LNMO magnetization curves (see Figures 5(b), 6(b), 7(b) and 8(b)) make evident that LCMO samples exhibit strong perpendicular magnetic anisotropy that is absent in LNMO samples. Worth to mention that LNMO samples show small in-plane anisotropy. Another interesting difference between $M(H)$ in LCMO and LNMO systems is the absence of APB in the later. The typical sudden drop of the magnetization at $\mathrm{H}=0$ observed very often in $\mathrm{M}(\mathrm{H})$ curves of LCMO 
samples, associated to the existence of APB, is completely absent in the case of LNMO samples. However, LNMO samples show clear evidences of ASD. The appearance of ASD, where $\mathrm{Ni}$ and $\mathrm{Mn}$ ions interchange their crystallographic sites, would mask the effect of multiple nucleation sites generating zones with alternated $\mathrm{Ni} / \mathrm{Mn}$ local ordering, thus precluding the formation of APB.
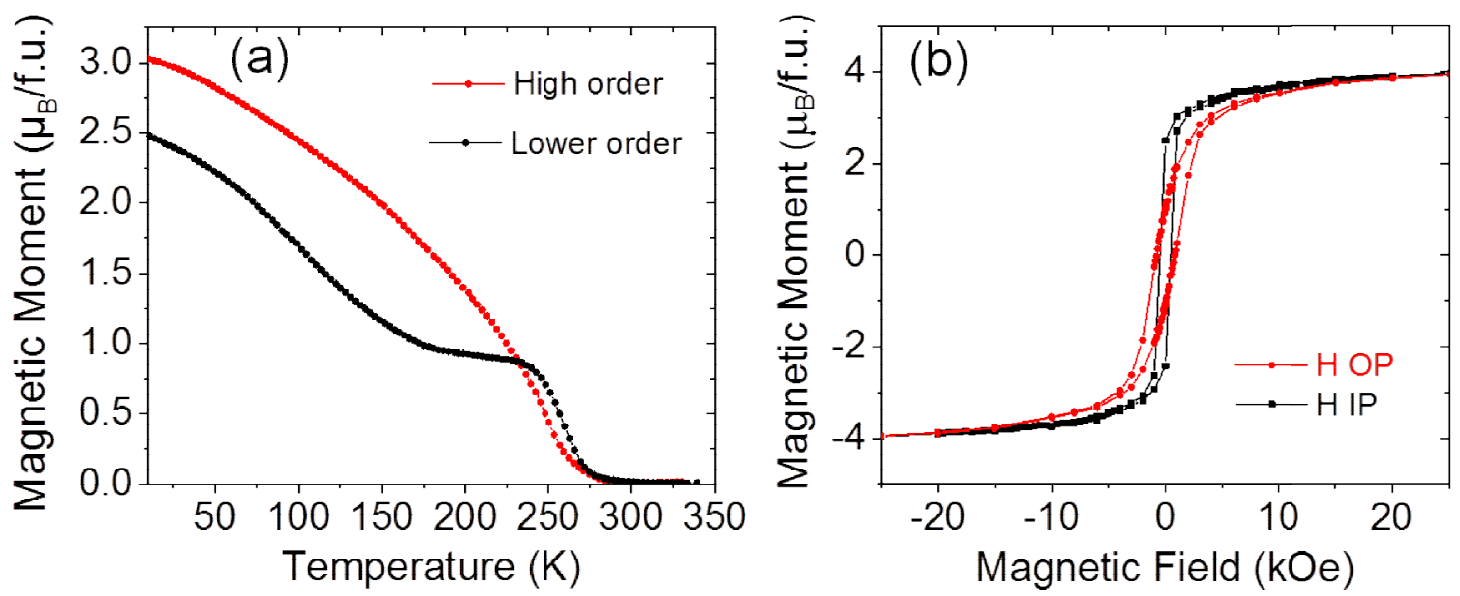

Figure 8: (a) Magnetization vs. temperature of two LNMO/LAO films with different amount of ASD, measured after field cooling with a magnetic field of $1 \mathrm{kOe}$ applied in-plane. (b) $\mathrm{M}-\mathrm{H}$ loops of the more ordered LNMO/LAO film in (a) recorded at $10 \mathrm{~K}$ by applying a magnetic field $\mathrm{H}$ parallel to (100)-LAO, in-plane; and parallel to (001)-LAO, out of plane.

On the other hand, the obtained values of $M_{S}=\left(1-2 X_{A S D}\right) M s \sim 4 \mu \mathrm{B} / f$.u., corresponds to a concentration of ASD of about $10 \%$ which are among the best in the literature. ${ }^{[31 b, 31 c, 32]}$ Therefore, we should conclude that the particular growth conditions of PAD technique promote a high degree of spontaneous B-site cationic ordering.

A way to discard the existence of secondary phases in our samples is to determine the oxidation state of $\mathrm{Ni}$ and $\mathrm{Mn}$ ions. For that purpose, an x-ray absorption spectroscopy (XAS) study has been performed. XAS measurements were done at the $\mathrm{Ni}$ and $\mathrm{Mn} \mathrm{L}_{2,3}$ edges in BL29-BOREAS beamline at ALBA Synchrotron Light Source (Barcelona, Spain). This technique is a very sensitive local probe, ideal to study the valence character of the ions under analysis ${ }^{[33]}$ and spin. ${ }^{[34]}$ 
(a)

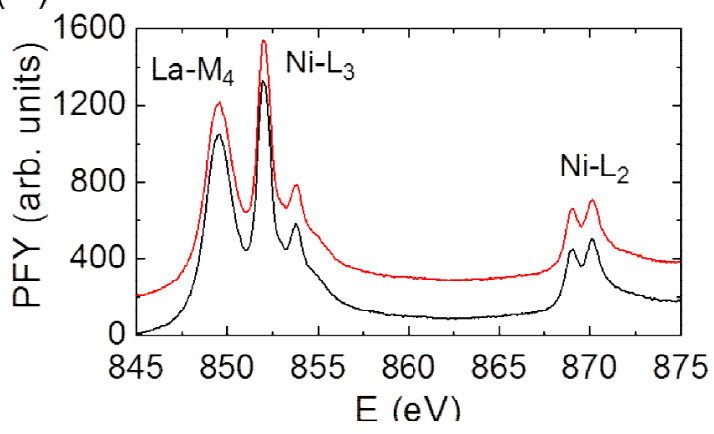

(b)

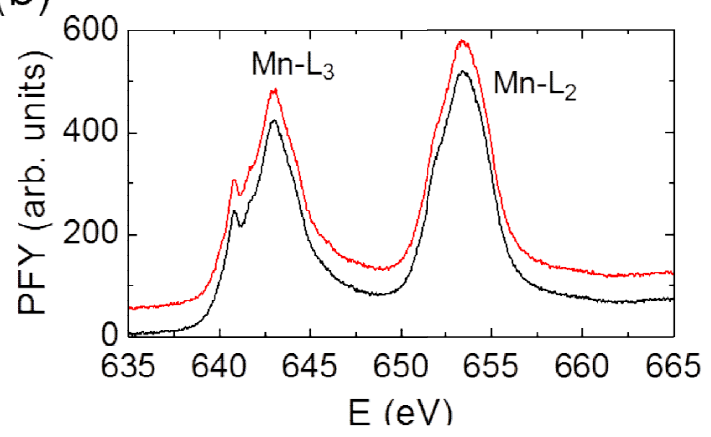

Figure 9: Partial fluorescence yield (PFY), of $\mathrm{Ni}_{2,3}$ and $\mathrm{Mn}_{2,3}$ edges measured in the two LNMO/STO thin films displayed in Fig. 7, with different amount of ASD. Red line high ordered sample, black line lower ordered sample.

Figure 9 shows the partial fluorescence yield (PFY) spectra corresponding to two LNMO samples with different amount of ASD (see Fig. 7) in the energy range 845-875 eV. As indicated La-M $\mathrm{M}_{4}$, and $\mathrm{Ni}-\mathrm{L}_{2,3}$ absorption edges are in this range of energies. Two important features can be identified for $\mathrm{Ni}$ edges. First, the two maxima of $\mathrm{Ni}-\mathrm{L}_{3}$ edge have very different heights and second, there is a clear split of $\mathrm{Ni}-\mathrm{L}_{2}$ edge (see Figure 9(a)). These two features have been identified as evidences of

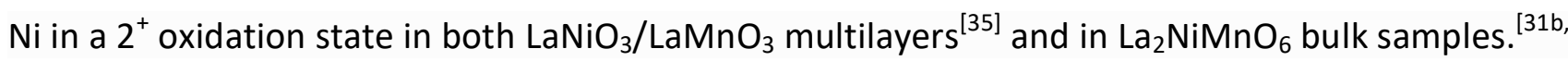
${ }^{31 c]}$ Figure 9 (b) shows the absorption of the same sample in the region corresponding to $M n-L_{2,3}$ edges. The position of $L_{3}$ maxima (643 eV) coincides quite well with that reported in Ref. 31b. for bulk samples of LNMO in which the valence of $\mathrm{Mn}$ is identified as $4^{+}$. Moreover, the feature visible at $640.8 \mathrm{eV}$ was identified with a strong $\mathrm{d}^{3}$ character of $\mathrm{Mn}$ electronic state $\left(\mathrm{Mn}^{4+}\right){ }^{[36]}$ As evidenced by the figure, spectra corresponding to samples with dofferent amount of ASD are very much alike. Therefore, XAS measurements confirm that the formal oxidation state of $\mathrm{Ni}$ and $\mathrm{Mn}$ ions in or samples is $\mathrm{Ni}^{2+}, \mathrm{Mn}^{4+}$, irrespective to the structural strain, and in spite of the different value of the saturation magnetization, i.e. the amount of ASD, in agreement with previous reports. So, we can conclude that the features observed in $M(T)$ curves in the LNMO system are not due to the existence o different magnetic phases but to different amount of disorder.

The final amount of ASD in a given sample can be influenced by several factors including oxygen and cationic non-stoichiometry, and synthesis and processing conditions. ${ }^{[37]}$ From a material synthesis standpoint, it has been shown that a large difference between the ionic size and electronegativity of the $\mathrm{B} / \mathrm{B}^{\prime}$ cations is crucial to promote ordering. ${ }^{[1]} \mathrm{XAS}$ analysis allows concluding that in both LCMO and LNMO systems the oxidation states of $\mathrm{Co}, \mathrm{Ni}$ and $\mathrm{Mn}$ are $\mathrm{Co}^{2+}, \mathrm{Ni}^{2+}$ and 
$\mathrm{Mn}^{4+}$, therefore no mayor differences are expected from this aspect. However, the ionic radii difference between $\mathrm{Co}^{2+}$ and $\mathrm{Mn}^{4+}$ is larger than that between $\mathrm{Ni}^{2+}$ and $\mathrm{Mn}^{4+}{ }^{[38]}$ (21.5 pm and 16.0 pm respectively) which makes B-site cationic ordering in LCMO system more favorable than for LNMO.

\section{Conclusion}

We have synthesized high quality $\mathrm{La}_{2} \mathrm{CoMnO}_{6}$ and $\mathrm{La}_{2} \mathrm{NiMnO}_{6}$ epitaxial thin films on STO and LAO substrates by polymer assisted deposition technique. Physical properties of these oxide compounds, in particular magnetic properties, are very sensitive to the existence of long-range Bsite cationic ordering; therefore, obtaining full cationic ordering is fundamental for potential technological applications. In this work, we show that the particular growth process and crystallization conditions of PAD (very slow rate, close to thermodynamic equilibrium conditions) promote the high crystallinity and quality of the films, as well as favors high $B$-site cationic ordering.

The magnetic properties of $\mathrm{La}_{2} \mathrm{CoMnO}_{6}$ films (Msat $\approx 5.9 \mu_{\mathrm{B}} / \mathrm{f}$.u. and $T c \approx 230 \mathrm{~K}$ ) are indicative of almost full Co/Mn B-site cationic ordering. However, in some cases signals of the existence of antiphase boundaries are detected. In the case of $\mathrm{La}_{2} \mathrm{NiMnO}_{6}$ epitaxial thin films superexchange interactions are a bit stronger leading to a ferromagnetic transition temperature of $T c \approx 260 \mathrm{~K}$. However, saturation magnetization values are depleted compared to the expected spin only theoretical value (Ms $=5 \mu_{\mathrm{B}} / \mathrm{f}$.u.) which suggest the existence of antisite disorder at the $\mathrm{Ni} / \mathrm{Mn}$ sublattice. Values around $M_{S} \sim 4 \mu_{B} / f . u$ are obtained indicating around $10 \%$ of ASD which compares very well with previous reports. The observed differences between LCMO and LNMO are attributed to the ionic radii difference between $\mathrm{Co}^{2+}$ and $\mathrm{Mn}^{4+}$ and that of $\mathrm{Ni}^{2+}$ and $\mathrm{Mn}^{4+}$ making $\mathrm{B}$ site cationic ordering in LCMO system more favorable than for LNMO.

Using $\mathrm{x}$-ray absorption spectroscopy technique, we have verified that the oxidation state of $\mathrm{Ni}$ is +2 , $\mathrm{Co}$ is $2+$ and that of $\mathrm{Mn}$ is +4 , irrespective to the structural strain and the amount of ASD of the samples. We conclude that the reduction of magnetic moment in LNMO samples is linked to the introduction of $\mathrm{Ni}^{2+}-\mathrm{O}^{2-}-\mathrm{Ni}^{2+}$ and $\mathrm{Mn}^{4+}-\mathrm{O}^{2-}-\mathrm{Mn}^{4+}$ superexchange $\mathrm{AF}$ interactions at antisites reducing the saturation magnetization, $M_{S}$, by a factor $\left(1-2 X_{A S D}\right)$, being $X_{A S D}$ the fraction of ASD disorder. 
Our results make evident the advantage of the CSD-PAD method over physical methods (far from thermodynamic equilibrium growth) to optimize spontaneous B-site cationic ordering as well as the desirable physical properties of functional double perovskite oxide thin films. In addition, in the context where the demand for sustainable and environmentally friendly reactants and processes steadily increases, PAD is based in aqueous solutions of environmentally friendly metal salts and commercially available polymers.

\section{Experimental Section}

\section{Chemicals and solvents}

The PAD technique relies on the use of water-soluble polymers with functional $-\mathrm{NH}_{2}$ groups that coordinate cations and prevent their hydrolysis. The precursor solution for the growth of the LCMO and LNMO thin films were prepared by mixing three separate aqueous solutions of La, Co, Mn and $\mathrm{La}, \mathrm{Ni}, \mathrm{Mn}$ respectively bound to polymers. Separate solutions were prepared using high-purity (>99.9\%) metal salts of lanthanum(III) nitrate, cobalt(II) nitrate, nickel(II) chloride, and $\mathrm{Mn}$ (II) nitrate. The polymer used was branched PEI, average $\mathrm{Mw} \sim 25,000$, and EDTA was used as a complexing agent; both were from Sigma Aldrich. Water used in the solution preparation was purified using a Milli-Q water treatment system.

\section{Precursor solutions preparation}

In detail, individual solutions of the different metal ions were prepared by dissolving the corresponding nitrate salts in water and EDTA (1:1 molar ratio). PEI was incorporated into the solution in a 1:1 mass ratio with EDTA. The electrostatic interactions of the protonated amino groups of PEI with the [EDTA-Metal] ${ }^{\mathrm{n}-}$ complex are crucial for further successful deposition of an homogeneous film. Since the direct bonding between PEI and metal is too weak, the chelate ligand EDTA is used to form the EDTA-metal complexes which can establish hydrogen bonding and electrostatic interactions with protonated $\mathrm{PEI}$. $\mathrm{pH}$ value plays important role in the formation of stable individual solutions of different metals. The enhancement of electrostatic interactions, particularly hydrogen bonds, takes places at lower values of $\mathrm{pH}$, where most of amines of PEI are protonated. The formation of more stable EDTA-metal complexes prefers higher values of $\mathrm{pH}$, while the hydrolysis of some cations occurs at relatively high $\mathrm{pH}$. For instance, a $\mathrm{pH} \approx 7$ is enough to hydrolyze $\mathrm{Mn}^{2+}$. The $\mathrm{pH}$ values of all the initial solutions for each individual cation in this work were 
adjusted to near 6 to balance all these situations, and prevent the precipitation when mixing them to prepare the final multicationic solutions.

Subsequently, the non coordinated cations of each single metal solution can be easily removed by filtration processes to prepare a homogeneous precursor solution for each single cation. Each individual solution was filtrated using Amicon ${ }^{\circledR}$ filtration units $(10 \mathrm{kDa})$, and retained portions were analyzed by inductively coupled plasma (ICP) (Optima 4300 ${ }^{\mathrm{TM}}$ DV ICP-OES Perkin-Elmer), for careful determination of the cation concentration in the primary solutions. The final concentrations of the solutions used in this work were $[\mathrm{La}]=230.4 \mathrm{mM},[\mathrm{Co}]=146.4 \mathrm{mM},[\mathrm{Ni}]=272.6 \mathrm{mM}$, and $[\mathrm{Mn}]=$ 176.4 mM. From these primary solutions, the final aqueous precursor solutions with the desired La:Co:Mn 2:1:1, and La:Ni:Mn 2:1:1 final stoichiometry were prepared, and concentrated to reach a final cation concentration of $61-65 \mathrm{mM}$ with respect to $\mathrm{Mn}$. These conditions were adjusted to produce films in the range of $16-26 \mathrm{~nm}$. Similarly, more diluted solutions were adjusted to obtain films in the range of a few $\mathrm{nm}(3-5 \mathrm{~nm})$. Typical viscosity values were in the range $\eta \approx 3-4 \mathrm{mPa} s$ (measured with a DMA 4100M Anton Paar densimeter, with a microviscometer module Lovis 2000 ME). Thermal analysis experiments were carried out with a Mettler Toledo TGA85 ${ }^{\mathrm{L}} \mathrm{LF}$ thermobalance. Complementary experiments were carried out with a differential scanning calorimeter (DSC) from TA Instruments (Q2000 apparatus).

\section{Thin films growth}

The precursor solutions obtained in this way were spin coated on top of $0.5 \times 0.5 \mathrm{~cm}^{2}$ (001)-STO and $0.5 \times 0.5 \mathrm{~cm}^{2}$ (001)-LAO substrates from Crystec, GmbH, Germany. Prior to deposition, the asreceived STO substrates were chemically etched and thermally treated to create $\mathrm{TiO}_{2}$-terminated substrates with atomically flat terraces, ${ }^{[39]}$ and the LAO substrates were thermally treated to create $\mathrm{AlO}_{2}$-terminated substrates with atomically flat terraces.

The last step is the thermal annealing of the spin coated films under oxygen flow for elimination of the organic components at lower temperatures, and phase formation and crystallization at higher temperatures. Thermal annealing was performed using a tube furnace with heating rates of several degrees Celsius per minute, under oxygen flow (flow rates between 100 and $600 \mathrm{ml} / \mathrm{min}$ ) to avoid the formation of oxygen vacancies. Good quality samples were obtained for optimized growth conditions (growth temperatures above $850^{\circ} \mathrm{C}$, annealing time of $\sim 1 \mathrm{~h}$, and oxygen flow above $0.3 \mathrm{l} / \mathrm{min})$. 


\section{Characterization of structural and physical properties}

The structural properties of the epitaxial films were studied by X-ray diffraction and reflectivity using a D5000 (Siemens) diffractometer, an X'Pert MRD (PANalytical) four-angle diffractometer with monochromatic $\mathrm{Cu}-\mathrm{K}_{\alpha 1}$ radiation (1.54060 ̊) and a Bruker D8 Advance GADDS system. Magnetization measurements were performed using a superconducting quantum interference device (SQUID; Quantum Design) as a function of temperature and magnetic field. External magnetic fields were applied both parallel (IP configuration) and perpendicular (OP configuration) to the sample plane. The diamagnetic contribution of the substrate and other instrumental contributions were properly corrected. ${ }^{[40]}$ The relative error in the determination of the saturation magnetization (Msat) was approximately $5 \%$ and was mostly attributable to the error in the estimation of the film volume. The surface topography of the films was investigated by atomic force microscopy (AFM) using an Asylum Research MFP-3D microscope in tapping mode.

X-ray absorption spectroscopy (XAS) and X-ray magnetic circular dichroism (XMCD) were investigated at the $\mathrm{Ni}$ and $\mathrm{Mn} \mathrm{L}_{2,3}$ edges in BL29-BOREAS beamline at ALBA Synchrotron Light Source (Barcelona, Spain). The spectra were measured in both the total electron yield (TEY) mode and by partial fluorescence yield (PFY), under ultrahigh vacuum conditions ( $\left.2 \times 10^{-10} \mathrm{mbar}\right)$. PFY was measured using a large area silicon drift detector collecting the non-resonant emission of O K-edge (energy window of about $100 \mathrm{eV}$ ) and applying the inverse partial fluorescence yield technique. ${ }^{[41]}$ The applied magnetic field (parallel to the $x$-ray beam) was $2 T$. All measures have been done at $\mathrm{T}=150 \mathrm{~K}$.

\section{Acknowledgements}

We acknowledge financial support from the Spanish Ministry of Science, Innovation and Universities through Severo Ochoa (SEV-2015-04969), HETEROCS (MAT2015-71664-R), SUMATE (RTI2018-095853-B-C21) and SPINCURIOX (RTI2018-099960-B-I00) projects cofinanced by the European Regional Development Fund. Hailin Wang acknowledges financial support from the China Scholarship Council (CSC). This work has been performed in the framework of the PhD programme in Materials Science of the Universitat Autònoma de Barcelona, through the China Scholarships Council (CSC)/Universitat Autònoma de Barcelona (UAB) Joint Scholarship. Authors thank ALBA Synchrotron (Spain) for the provision of beamtime. Authors also thank Dr. B. Bozzo and F. J. Campos for their technical support during magnetic and X-ray diffraction measurements.

Conflict of interest The authors declare no conflict of interest. 
Preprint of the paper pulished in Chemistry - A European Journal, 2020, 26, 9338-9347; doi.org/10.1002/chem.202000129

\section{Keywords:}

chemical growth methods, water-based precursor solutions, polymer assisted deposition, double perovskites, B-site ordering, ferromagnetism

\section{REFERENCES:}

[1] a) M. T. Anderson, K. B. Greenwood, G. A. Taylor, K. R. Poeppelmeier, Prog. Solid State Chem. 1993, 22, 197-233; b) S. Vasala, M. Karppinen, Prog. Solid State Chem. 2015, 43, 1-36.

[2] a) K. I. Kobayashi, T. Kimura, H. Sawada, K. Terakura, Y. Tokura, Nature 1998, 395, 677-680; b) Y. Moritomo, S. Xu, A. Machida, T. Akimoto, E. Nishibori, M. Takata, M. Sakata, Phys. Rev. B 2000, 61, R7827-R7830; c) M. Garcia-Hernandez, J. L. Martinez, M. J. Martinez-Lope, M. T. Casais, J. A. Alonso, Phys. Rev. Lett. 2001, 86, 2443-2446; d) A. T. Lee, C. A. Marianetti, Phys. Rev. B 2018, 97, 045102.

[3] a) G. Blasse, J. Phys. Chem. Solids 1965, 26, 1969-1971; b) G. H. Jonker, J. Appl. Phys. 1966, 37, 14241430.

[4] a) J. B. Goodenough, Phys. Rev. 1955, 100, 564-573; b) J. Kanamori, J. Phys. Chem. Solids 1959, 10, 87-98.

[5] a) R. I. Dass, J. B. Goodenough, Phys. Rev. B 2003, 67, 014401; b) R. I. Dass, J. Q. Yan, J. B. Goodenough, Phys. Rev. B 2003, 68, 064415.

[6] M. P. Singh, K. D. Truong, P. Fournier, Appl. Phys. Lett. 2007, 91, 042504.

[7] R. N. Mahato, K. Sethupathi, V. Sankaranarayanan, J. Appl. Phys. 2010, 107, 09 D714.

[8] L. López-Mir, C. Frontera, H. Aramberri, K. Bouzehouane, J. Cisneros-Fernandez, B. Bozzo, L. Balcells, B. Martinez, Sci. Rep. 2018, 8, 861.

[9] C. Lan, S. Zhao, T. Xu, J. Ma, S. Hayase, T. Ma, J. Alloys Compd. 2016, 655, 208-214

[10] L.-P. Sun, H. Li, Q. Li, L.-H. Huo, H. Zhao, J.-M. Bassat, A. Rougier, S. Fourcade, J.-C. Grenier, J. Power Sources 2018, 392, 8-14.

[11] a) A. Y. Cho, J. R. Arthur, Prog. Solid State Chem. 1975, 10, 157-191; b) D. G. Schlom, APL Mater. 2015, 3, 062403.

[12] a) I. Safi, Surf. Coat. Techol. 2000, 127, 203-218; b) R. Galceran, C. Frontera, L. Balcells, J. CisnerosFernández, L. López-Mir, J. Roqueta, J. Santiso, N. Bagués, B. Bozzo, A. Pomar, F. Sandiumenge, B. Martínez, Appl. Phys. Lett. 2014, 105, 242401.

[13] a) D. H. A. Blank, G. Koster, G. A. J. H. M. Rijnders, S. Eelco van, P. Slycke, H. Rogalla, J. Cryst. Growth 2000, 211, 98-105; b) M. Opel, J. Phys. D: Appl. Phys. 2012, 45, 033001.

[14] a) R. W. Schwartz, T. Schneller, R. Waser, C. R. Chim. 2004, 7, 433-461; b) T. Schneller, R. Waser, M. Kosec, D. Payne, 1 ed., Springer-Verlag Wien, 2013, pp. 1-796; c) M. L. Calzada, in The Sol-Gel Handbook (Ed.: D. L. M. Zayat), Wiley - VCH Verlag GmbH \& Co. KGaA, 2015, pp. 841-882; d) J. E. ten Elshof, in Epitaxial Growth of Complex Metal Oxides, 1 ed., Woodhead Publishing, 2015, pp. 69-93; e) N. Bassiri-Gharb, Y. Bastani, A. Bernal, Chem. Soc. Rev. 2014, 43, 2125-2140.

[15] a) M. G. Kim, M. G. Kanatzidis, A. Facchetti, T. J. Marks, Nat. Mater. 2011, 10, 382-388; b) P.-C. Wu, Y.-H. Chu, J. Mater. Chem. C 2018, 6, 6102-6117; c) I. Bretos, R. Jimenez, J. Ricote, M. L. Calzada, Chem. Soc. Rev. 2018, 47, 291-308.

[16] I. Bretos, R. Jimenez, D. Perez-Mezcua, N. Salazar, J. Ricote, M. L. Calzada, Adv. Mater. 2015, 27, 2608-2613.

[17] G. Wulfsberg, Principles of descriptive inorganic chemistry, Brooks/Cole Pub. Co., Monterey, Calif., 1987.

[18] Q. X. Jia, T. M. McCleskey, A. K. Burrell, Y. Lin, G. E. Collis, H. Wang, A. D. Li, S. R. Foltyn, Nat. Mater. 2004, 3, 529-532.

[19] a) A. K. Burrell, T. Mark McCleskey, Q. X. Jia, Chem. Commun. 2008, 1271-1277; b) G. F. Zou, J. Zhao, H. M. Luo, T. M. McCleskey, A. K. Burrell, Q. X. Jia, Chem. Soc. Rev. 2013, 42, 439-449. 
[20] J. M. Vila-Fungueiriño, B. Rivas-Murias, J. Rubio-Zuazo, A. Carretero-Genevrier, M. Lazzari, F. Rivadulla, J. Mater. Chem. C 2018, 6, 3834-3844.

[21] H. Wang, J. Gazquez, C. Frontera, M. F. Chisholm, A. Pomar, B. Martinez, N. Mestres, NPG Asia Mater. 2019, 11, 44.

[22] M. K. Kim, J. Y. Moon, H. Y. Choi, S. H. Oh, N. Lee, Y. J. Choi, J. Phys. Condens. Matter 2015, 27, 426002.

[23] H. Guo, J. Burgess, S. Street, A. Gupta, T. G. Calvarese, M. A. Subramanian, Appl. Phys. Lett. 2006, 89, 022509.

[24] a) R. Galceran, L. López-Mir, B. Bozzo, J. Cisneros-Fernández, J. Santiso, L. Balcells, C. Frontera, B. Martínez, Phys. Rev. B 2016, 93, 144417; b) L. López-Mir, R. Galceran, J. Herrero-Martín, B. Bozzo, J. Cisneros-Fernández, E. V. Pannunzio Miner, A. Pomar, L. Balcells, B. Martínez, C. Frontera, Phys. Rev. B 2017, 95, 224434.

[25] L. Balcells, J. Navarro, M. Bibes, A. Roig, B. Martínez, J. Fontcuberta, Appl. Phys. Lett. 2001, 78, 781783.

[26] H. Z. Guo, A. Gupta, J. Zhang, M. Varela, S. J. Pennycook, Appl. Phys. Lett. 2007, 91, 202509.

[27] A. J. Baron-Gonzalez, C. Frontera, J. L. Garcia-Munoz, B. Rivas-Murias, J. Blasco, J. Phys. Condens. Matter 2011, 23, 496003.

[28] a) H. Z. Guo, A. Gupta, T. G. Calvarese, M. A. Subramanian, Appl. Phys. Lett. 2006, 89, 262503; b) R. Egoavil, S. Huhn, M. Jungbauer, N. Gauquelin, A. Beche, G. Van Tendeloo, J. Verbeeck, V. Moshnyaga, Nanoscale 2015, 7, 9835-9843.

[29] a) K. D. Truong, M. P. Singh, S. Jandl, P. Fournier, Phys. Rev. B 2009, 80, 134424 ; b) M. P. Singh, C. Grygiel, W. C. Sheets, P. Boullay, M. Hervieu, W. Prellier, B. Mercey, C. Simon, B. Raveau, Appl. Phys. Lett. 2007, 91, 012503; c) M. P. Singh, S. Charpentier, K. D. Truong, P. Fournier, Appl. Phys. Lett. 2007, 90, 211915.

[30] a) V. L. J. Joly, P. A. Joy, S. K. Date, C. S. Gopinath, Phys. Rev. B 2002, 65, 184416; b) J. Blasco, M. C. Sánchez, J. Pérez-Cacho, J. García, G. Subías, J. Campo, J. Phys. Chem. Solids 2002, 63, 781-792.

[31] a) D. Choudhury, P. Mandal, R. Mathieu, A. Hazarika, S. Rajan, A. Sundaresan, U. V. Waghmare, R. Knut, O. Karis, P. Nordblad, D. D. Sarma, Phys. Rev. Lett. 2012, 108, 127201; b) S. Pal, G. Sharada, M. Goyal, S. Mukherjee, B. Pal, R. Saha, A. Sundaresan, S. Jana, O. Karis, J. W. Freeland, D. D. Sarma, Phys. Rev. B 2018, 97, 165137; c) M. Nasir, S. Kumar, N. Patra, D. Bhattacharya, S. N. Jha, D. R. Basaula, S. Bhatt, M. Khan, S.-W. Liu, S. Biring, S. Sen, ACS Appl. Electron. Mater. 2019, 1, 141-153.

[32] a) S. R. Spurgeon, Y. Du, T. Droubay, A. Devaraj, X. Sang, P. Longo, P. Yan, P. G. Kotula, V. Shutthanandan, M. E. Bowden, J. M. LeBeau, C. Wang, P. V. Sushko, S. A. Chambers, Chem. Mater. 2016, 28, 3814-3822; b) M. Nasir, M. Khan, S. Kumar, S. Bhatt, N. Patra, D. Bhattacharya, S. N. Jha, S. Biring, S. Sen, J. Magn. Magn. Mater. 2019, 483, 114-123.

[33] C. Mitra, Z. Hu, P. Raychaudhuri, S. Wirth, S. I. Csiszar, H. H. Hsieh, H. J. Lin, C. T. Chen, L. H. Tjeng, Phys. Rev. B 2003, 67, 092404.

[34] S. Ray, A. Kumar, D. D. Sarma, R. Cimino, S. Turchini, S. Zennaro, N. Zema, Phys. Rev. Lett. 2001, 87, 097204.

[35] C. Piamonteze, M. Gibert, J. Heidler, J. Dreiser, S. Rusponi, H. Brune, J. M. Triscone, F. Nolting, U. Staub, Phys. Rev. B 2015, 92, 014426.

[36] M. Abbate, F. M. de Groot, J. C. Fuggle, A. Fujimori, O. Strebel, F. Lopez, M. Domke, G. Kaindl, G. A. Sawatzky, M. Takano, Y. Takeda, H. Eisaki, S. Uchida, Phys. Rev. B 1992, 46, 4511-4519.

[37] M. P. Singh, K. D. Truong, S. Jandl, P. Fournier, J. Appl. Phys. 2010, 107, 09 D917.

[38] R. D. Shannon, Acta Cryst. A 1976, 32, 751-767.

[39] M. Kareev, S. Prosandeev, J. Liu, C. Gan, A. Kareev, J. W. Freeland, M. Xiao, J. Chakhalian, Appl. Phys. Lett. 2008, 93, 061909.

[40] P. Stamenov, J. M. D. Coey, Rev. Sci. Instrum. 2006, 77, 015106.

[41] A. J. Achkar, T. Z. Regier, H. Wadati, Y. J. Kim, H. Zhang, D. G. Hawthorn, Phys. Rev. B 2011, 83, 081106(R). 